\title{
A IMPORTÂNCIA E OS DESAFIOS DA ESTRATÉGIA DE SAÚDE DA FAMÍLIA NO ENFRENTAMENTO DA COVID-19
}

\section{Romana Erica Tavares Grangeiro Pinto후 Wyara Ferreira Melo²; Maria Amanda Laurentino Freires $^{3}$; Mônica Valéria Barros Pereira ${ }^{4}$; Aline Carla de Medeiros ${ }^{5}$; José Cândido da Silva Nóbrega $^{6}$; Manoel Marques de Souto Nóbrega Filho ${ }^{7}$; Vicente Saraiva dos Santos Neto ${ }^{8}$; Patrício Borges Maracajá9.}

${ }^{1}$ Faculdade Santa Maria (FSM), Cajazeiras, Paraíba.

${ }^{2}$ Universidade Federal de Campina Grande (UFCG), Campina Grande, Paraíba. ${ }^{3}$ Faculdade Santa Maria (FSM), Cajazeiras, Paraíba. ${ }^{4}$ Centro Universitário de João Pessoa (UNIPE), Paraíba.

${ }^{5}$ Universidade Federal de Campina Grande (UFCG), Campina Grande, Paraíba. ${ }^{6}$ Instituto de Educação Superior da Paraíba (IESP), Pombal, Paraíba.

${ }^{7}$ Faculdade de Ciências Médicas da Paraíba (FCM-PB), João Pessoa, Paraíba. ${ }^{8}$ Faculdade Santa Maria, Cajazeiras, Paraíba.

${ }^{9}$ Universidade Federal de Campina Grande (UFCG), Campina Grande, Paraíba.

\section{DOI: 10.47094/IICNNESP.2021/28}

\section{RESUMO}

O estudo objetiva conhecer através da revisão bibliográfica a importância e os possíveis desafios da Estratégia de Saúde da Família no enfrentamento da COVID-19. Trata-se de uma revisão bibliográfica, com abordagem exploratória, realizada através das bases de dados da LILACS e na SciELO. Descritores utilizados: Atenção Primária. Pandemia. Saúde Pública. O desenvolvimento do estudo fundamenta-se apresentando os aspectos gerais sobre a pandemia do COVID-19 e a importância e desafios da Estratégia de Saúde da Família no enfrentamento da COVID-19. Concluise que é extremamente necessário que mais estudos possam ser realizados e divulgados, para que cada vez mais a população, em geral, tenha acesso à notícias reais e que esta tenha consciência do papel não somente dos profissionais de saúde ou das políticas públicas de saúde, como também da sua responsabilidade individual e coletiva para o enfrentamento a pandemia do COVID-19.

PALAVRAS-CHAVE: Atenção Primária. Pandemia. Saúde Pública.

ÁREA TEMÁTICA: Política e gestão em Saúde. 


\section{INTRODUÇÃO}

A Atenção Básica $(\mathrm{AB})$ consolida-se como coordenadora do cuidado e ordenadora das ações e serviços disponibilizados na rede, visto que, os profissionais inseridos nas Unidade Básicas de Saúde (UBS) são responsáveis pelo acompanhamento do estado de saúde da população adstrita do seu território, tendo uma visão mais ampla dos usuários em risco de adoecimento ou agravamento de condições prévias para o COVID-19. Tal estratégia de saúde vêm demonstrando, ao longo de sua consolidação, resultados que denotam efetividade, levando em conta o marco conceitual de sistema de saúde da OMS, ampliando e oferecendo a população serviços de qualidade (CABRAL et al., 2020).

Partindo de tudo que foi abordado, a escolha pelo desenvolvimento do estudo emergiu da necessidade em abordar e discutir como a pandemia provocada pelo novo coronavírus Sars-Cov-2 afetou e vem afetando as organizações e os indivíduos em todas as escalas de suas vidas. Com isso, o tema além de ser extremamente atual, é também muito relevante nesse cenário em que rotinas foram alteradas e que se estabeleceu "um novo normal" na vida de todas as pessoas, ampliando assim a importância da saúde pública e da atenção básica para que a população receba o suporte necessário. Nesse sentido, a pesquisa tem um caráter não apenas acadêmico, mas também social, servindo como suporte para pesquisas posteriores e para a prática da equipe multiprofissional que compõe a estratégia de saúde da família.

Destarte, o estudo em questão tem como finalidade conhecer através da revisão bibliográfica a importância e os possíveis desafios da Estratégia de Saúde da Família no enfrentamento da COVID-19, buscando também compreender os aspectos gerais sobre a pandemia do COVID-19 e apresentar uma visão ampla a respeito da Estratégia de Saúde da Família.

\section{METODOLOGIA}

A presente pesquisa trata-se de uma revisão bibliográfica, com abordagem exploratória, realizada a partir da busca de artigos publicados e indexados nas bases de dados da Literatura Latino Americana e do Caribe em Ciências da Saúde (LILACS), na Scientific Eletronic Library Online (SciELO) e em outros periódicos confiáveis. Para a busca dos estudos elencados foram utilizados os seguintes descritores e suas combinações em língua portuguesa: Atenção Primária. Pandemia. Saúde Pública.

Os critérios de seleção definidos para seleção das publicações foram os seguintes: artigos científicos, monografias, dissertações e teses que apresentam a temática do estudo, que estão em língua inglesa e portuguesa, acesso gratuito, texto completo, publicados e indexados nos referidos bancos de dados entre os anos de 2018 a 2021. Foram excluídos da pesquisa os estudos que não permeassem os critérios de inclusão pré-estabelecidos. 


\section{FUNDAMENTAÇÃO TEÓRICA}

\section{Aspectos gerais sobre a pandemia do covid-19}

A pandemia pelo SARS-CoV-2 iniciou-se na cidade de Wuhan, região central da China no final do ano de 2019, a transmissão da doença estava relacionada ao mercado de frutos do mar e de animais vivos. Rapidamente se alastrou para toda China, a Ásia e, em dois meses, atingiu todos os continentes. Em 30 de janeiro de 2020, a Organização Mundial da Saúde (OMS) declarou Emergência de Saúde Pública de Importância Internacional (ESPII) em razão do espalhamento da COVID-19. Em 3 de fevereiro de 2020, o Ministério da Saúde do Brasil declarou Emergência de Saúde Pública de Importância Nacional (ESPIN), em decorrência da infecção humana pelo Covid-19, por meio da Portaria MS n¹88/2020 (FERREIRA; LINO, 2020).

A respeito da transmissão do SARS-CoV-2, Aquino et al. (2020) acrescentam que ela ocorre, predominantemente, por meio de gotículas contaminadas de secreções da orofaringe de uma pessoa infectada para uma pessoa livre da infecção, apesar de ainda ser desconhecido o papel da transmissão por aerossóis, pelo contato com superfícies e objetos contaminados, onde o vírus pode permanecer viável por até 72 horas, ou por via fecal-oral. A transmissão do SARS-CoV-2 é agravada pelo elevado tempo médio de incubação, de aproximadamente 5-6 dias (variando de 0 a 24 dias) 8-10, e devido a pessoas sem sintomas, pré-sintomáticas ou com sintomas leves poderem transmitir a doença.

\section{Importância e desafios da estratégia de saúde da família no enfrentamento da covid-19}

A reorganização dos serviços de APS para, simultaneamente, enfrentar a epidemia e manter a oferta regular de suas ações é imperativa, passando por um processo de protagonismo e readequação. Mesmo reconhecendo as diversas fragilidades de atuação das equipes, ressalta-se que a Estratégia Saúde da Família (ESF) é o modelo mais adequado por seus atributos de responsabilidade territorial e orientação comunitária, para apoiar as populações em situação de isolamento social pois, é preciso manter o contato e o vínculo das pessoas com os profissionais, responsáveis pelo cuidado à saúde (MEDINA et al., 2020).

Mediante essas discussões, Nedel (2020) elucida que a AB é a fortaleza do sistema, na clínica individual e familiar e ações comunitárias na identificação de casos, contribuindo assim com um muito maior controle da pandemia, promovendo o isolamento social através de ações de saúde comunitária.

De acordo com Cabral et al. (2020), é necessário pensar em estratégias conjuntas de enfrentamento da epidemia em curso, buscando estabelecer linhas de cuidado na rede local e intermunicipal de saúde, trabalho interprofissional, parcerias intersetoriais, melhoria da comunicação entre todos os níveis de atenção, fortalecimento do controle social, implementação de protocolos clínicos e de manejo de casos suspeitos e confirmados de COVID-19. Essa rede de cooperação deve estar voltada ao cuidado longitudinal, com vistas a um enfrentamento eficaz da pandemia, com o achatamento da curva de infecção e de mortalidade. 


\section{CONSIDERAÇÕES FINAIS}

Conclui-se que é extremamente necessário que mais estudos possam ser realizados e divulgados, para que cada vez mais a população, em geral, tenha acesso as notícias reais e que esta tenha consciência do papel não somente dos profissionais de saúde ou das políticas públicas de saúde, como também da sua responsabilidade individual e coletiva para o enfrentamento a pandemia do COVID-19.

\section{PRINCIPAIS REFERÊNCIAS}

AQUINO, Estela M. L. et al. Medidas de distanciamento social no controle da pandemia de COVID-19: potenciais impactos e desafios no Brasil. Ciência \& Saúde Coletiva, v. 25, supl.1, p. 2423-2446, 2020. Disponível em: <https://www.scielosp.org/pdf/csc/2020.v25suppl1/2423-2446/pt>. Acesso em 07 de janeiro de 2021.

BRASIL. Ministério da saúde. Secretaria de Vigilância em Saúde. Departamento de Imunização e Doenças Transmissíveis. Coordenação-Geral do Programa Nacional de Imunizações. Plano Nacional de Operacionalização da Vacinação contra a COVID-19. 2 ed. Brasília, 2021a. Disponível em: <http://www.saude.pi.gov.br/uploads/warning_document/file/641/Plano_Nacional_ de_Vacina\%C3\%A7\%C3\%A3o_Covid19.pdf>. Acesso em 13 de janeiro de 2021.

CABRAL, Elizabeth Regina de Melo et al. Contribuições e desafios da Atenção Primária à Saúde frente à pandemia de COVID-19. InterAm J Med Health, 2020. Disponível em: <https://iajmh. emnuvens.com.br/iajmh/article/view/87/130>. Acesso em 12 de fevereiro de 2021.

VALE, Thaynara Duarte do et al. COVID-19 e idoso: medidas de isolamento social e exacerbação da violência e abuso familiar. Braz. J. Hea. Rev, Curitiba, v. 3, n. 6, p.17344-17352, nov./dez., 2020.

MEDINA, Maria Guadalupe et al. Atenção primária à saúde em tempos de COVID-19: o que fazer? Cad. Saúde Pública, v. 36, n. 8, 2020. Disponível em: <https://www.scielo.br/pdf/csp/v36n8/16784464-csp-36-08-e00149720.pdf>. Acesso em 09 de fevereiro de 2021.

NEDEL, Fúlvio Borges. Enfrentando a COVID-19: APS: forte agora mais que nunca! APS em Revista, v. 2, n. 1, p. 11-16, Jan./Abr., 2020. Disponível em: <https://apsemrevista.org/aps/article/ view/68/42>. Acesso em 07 de fevereiro de 2021.

PEREIRA JUNIOR, Luciano Aparecido; BERETTA, Regina Celia de Souza. A Estratégia de Saúde da Família e o enfrentamento à violência e fatores de risco. Psicologia e Saúde em Debate, v. 5, n. 2, p. 82-95, Dez., 2019. Disponível em: <http://psicodebate.dpgpsifpm.com.br/index.php/periodico/ article/view/V5N2A6/402>. Acesso em 10 de fevereiro de 2021.

VALE, Thaynara Duarte do et al. COVID-19 e idoso: medidas de isolamento social e exacerbação da violência e abuso familiar. Braz. J. Hea. Rev, Curitiba, v. 3, n. 6, p.17344-17352, nov./dez., 2020. 